This is the pre-peer revied version of a paper published by Advanced Functional Materials (http://onlinelibrary.wiley.com/journal/10.1002/(ISSN)1616-3028) which can be obtained under: http://onlinelibrary.wiley.com/doi/10.1002/adfm.201100063/abstract

DOI: $10.1002 / \mathrm{adfm} .201100063$

\title{
Field-tuneable diamagnetism in ferromagnetic-superconducting core-shell structures.
}

By André Müller*, and Sara E. C. Dale, and Miles A. Engbarth, and Simon J. Bending*, and Laurence M. Peter, and Andreas Knittel, and Hans Fangohr.

[*] Corresponding Authors

A. Müller, Dr. S. E.C. Dale, Dr. M. A. Engbarth, Prof. S. J. Bending

Department of Physics

Claverton Down, Bath BA2 7AY, UK

E-mail: A.Mueller@Bath.ac.uk, S.Bending@Bath.ac.uk

Prof. L. M. Peter

Department of Chemistry

Claverton Down, Bath BA2 7AY, UK

A. Knittel, Dr. H. Fangohr

School of Engineering Sciences

University of Southampton, Southampton SO17 1BJ, UK

Keywords: Hybrid materials, Core/Shell Nanoparticles, Superconductors, Magnetic Materials, Data storage, Sensors

Three dimensional ferromagnet-superconductor core-shell structures are realized by electrocrystallisation and their magnetic properties investigated. We observe fully re-entrant core superconductivity in increasing fields that survives well above the bulk critical field due to compensation effects. The net measured magnetization of optimized structures could be switched from absolute paramagnetic to diamagnetic by tuning the external magnetic field. Micromagnetic simulations of our structures are in good qualitative agreement with our results. 


\section{Introduction}

The ability to controllably reverse the sign of magnetization of a material would lead to many novel applications in the areas of magnetic guidance and magnetic devices. The ultimate example of this would be switching a perfectly diamagnetic superconducting sample into a ferromagnetic one. Superconductivity and ferromagnetism are, however, generally mutually incompatible phenomena since the exchange interaction that leads to spin alignment in ferromagnets destroys the usual superconducting ground state composed of electron pairs with opposite spins. Superconductivity can, however, sometimes be induced in ferromagnetic metals by a large applied magnetic field that opposes and compensates the exchange field; the so-called Jaccarino-Peter (J-P) effect ${ }^{[1]}$ which is accompanied by the reversal of the sign of magnetization. The apparent conflict between the two phenomena can also be overcome in inhomogeneous materials when the carriers responsible for the different ordered states are separated in space. This principle has guided the development of artificial ferromagnetsuperconductor hybrid (FSH) structures that have been intensively investigated in recent years. $^{[2-5]}$

The combination of superconductors with magnetic materials leads to many interesting potential applications. If the stray fields of the ferromagnetic component are relatively weak they can partially compensate an externally applied magnetic field, or a field generated by a superconducting current, leading to an increase in the overall critical field(current) at which the superconducting state is destroyed. Alternatively, if the stray field of the ferromagnet exceeds the critical field in the adjacent superconductor, superconductivity can be switched on again by applying an opposing external field to compensate it. Such field-induced superconductivity can be thought of as kind of inhomogeneous analogue of the J-P effect and was recently demonstrated in thin $\mathrm{Pb}$ films with arrays of $\mathrm{Co} / \mathrm{Pd}$ nanomagnets patterned on the surface. ${ }^{[6]}$ Such FSH could find important applications in novel superconducting magnetic memory devices, ${ }^{[7-9]}$ or might be incorporated into superheated tin granules for high energy 
particle detection at much higher magnetic fields. ${ }^{[10]}$ In addition FSH composites could, in principle, be designed in such a way that their net magnetic response changes from para(ferro)magnetic to diamagnetic over a distinct range of externally applied fields (though this has not yet been demonstrated in lithographic samples to the best of our knowledge). The corresponding change in sign of the magnetic force exerted on the FSH could be exploited in advanced magnetic guidance devices.

To date, experimental and theoretical studies of FSH have focused primarily on one dimensional nanowires/nanorods, ${ }^{[1]}$ two dimensional layered structures ${ }^{[12-16]}$ or arrays of ferromagnetic nanodots patterned on top of thin superconducting films. $\left.{ }^{[6,17}-19\right]$ The linear/planar geometry of the ferromagnetic component in such structures typically leads to strong demagnetization effects and limits the scope for controlling the magnetic environment of the adjacent superconductor. Recent developments in electrocrystallisation have, however, made it possible to realize fully three dimensional core-shell structures combining various superconducting and ferromagnetic materials. ${ }^{[20-22]}$ In stark contrast to earlier work, where FSHs were often prepared using lithographic methods, the electrochemical approach allows one to prepare highly faceted three dimensional single crystalline superconducting 'cores' that are completely or mostly covered by a polycrystalline ferromagnetic 'shell'. In addition, our preparation method is much more suitable for practical applications, being faster and cheaper than lithographic approaches and readily scalable to large sample numbers.

Here we report Hall magnetometry on individual core-shell structures of this type where the core is a highly faceted microcrystal of superconducting lead $(\mathrm{Pb})$ or tin $(\mathrm{Sn})$ and the shell is composed of a ferromagnetic Ni film. We show that the magnetic properties of these FSH can be controlled by varying their size and shape as well as the thickness of the ferromagnetic shell. Optimized structures show fully re-entrant superconductivity at high magnetic fields whose measured magnetization completely reverses from para(ferro-)magnetic to diamagnetic over a fairly narrow range of applied fields. 
Our results not only demonstrate the advantages of using electrochemistry to prepare three dimensional FSHs, but also illustrate their fascinating new properties.

\section{Results}

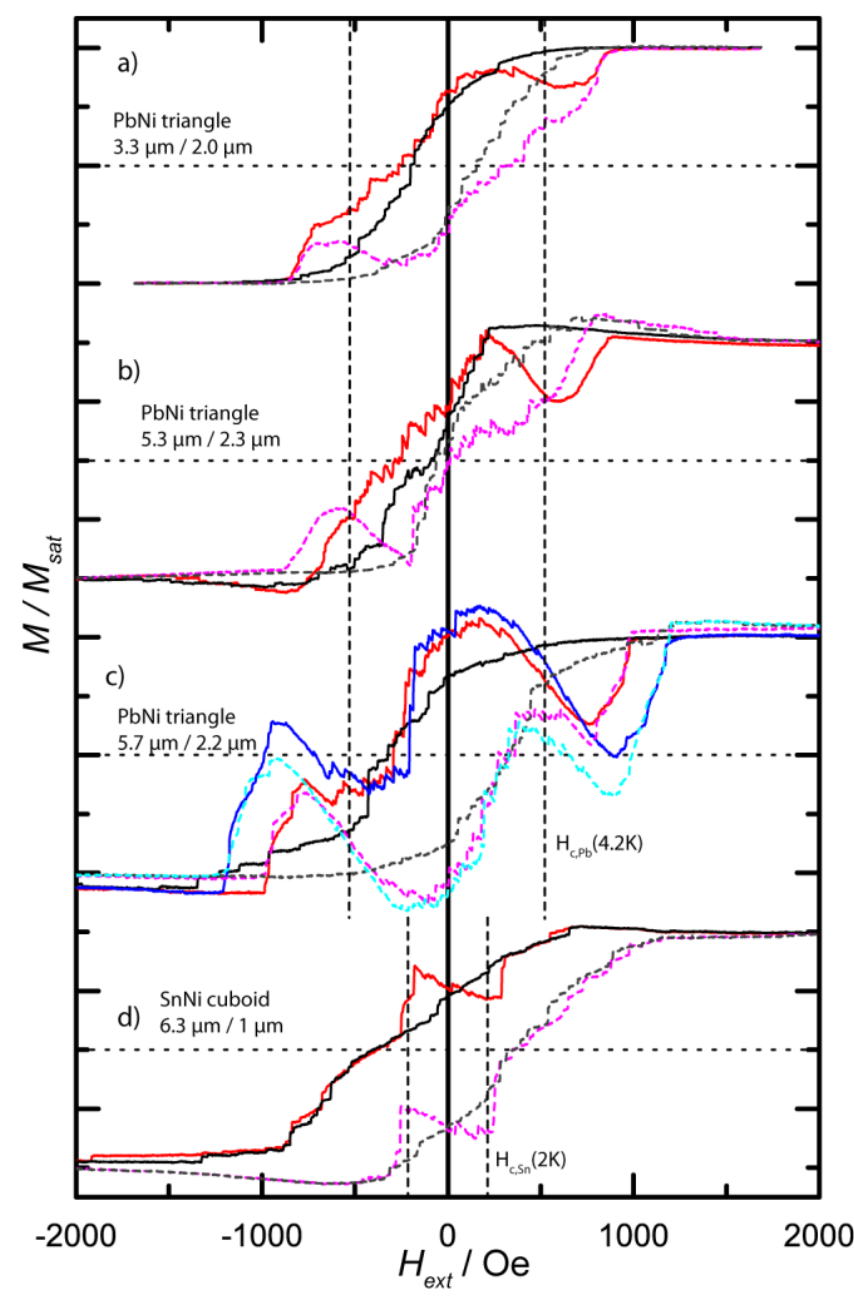

Figure 1.

Plots of $M / M_{\text {sat }}$ versus $H_{\text {ext }}$ for three different $\mathrm{Pb}-\mathrm{Ni}$ triangular-shaped samples (a - c) and a Sn-Ni square cuboid-shaped sample (d). Curves have been offset for clarity. For each measured FSH one curve for $T=4.2 \mathrm{~K}<T_{c}^{\mathrm{Pb}}\left(T=2 \mathrm{~K}<T_{c}^{\mathrm{Sn}}\right.$ for the Sn-Ni crystal in d $)$ is shown for positive (solid, red) and negative (dotted, purple) field sweeps, as well as a similar curve for $T>T_{c}^{\text {core }}$ (solid black and dotted dark grey respectively). For the sample in plot (c), an additional curve is shown at $T=2 \mathrm{~K}$ (solid, blue and dotted, turquoise) illustrating the absolute change from measured para(ferro-)magnetic to diamagnetic behavior at intermediate external fields.

Figure 1 shows 'local' magnetization data measured for four different FSH crystals. The three top curves (a-c) show data for $\mathrm{Pb}-\mathrm{Ni}$ triangular platelet-shaped crystals, while the bottom 
curve (d) shows data for a Sn-Ni cuboid-shaped crystal. For each crystal the pure ferromagnetic signal from the $\mathrm{Ni}$ shell measured at a temperature above the critical temperature of the core is shown in dark grey and black for up and down field sweeps respectively. The red (down sweep) and magenta (up sweep) curves show the local magnetization for $T=4.2 \mathrm{~K}<T_{c}^{\mathrm{Pb}}=7.18 \mathrm{~K}$ for the $\mathrm{Pb}$-Ni samples and for $T=2 \mathrm{~K}<T_{c}^{\mathrm{Sn}}=$ 3.72 K for the Sn-Ni sample. Clearly the measured magnetization changes dramatically when the core becomes superconducting. The critical field of bulk lead $\left(H_{c}{ }^{\mathrm{Pb}}(4.2 \mathrm{~K})=527 \mathrm{G}\right)$ is indicated by vertical dotted lines for comparison and we see that superconductivity survives well above this field in all three $\mathrm{Pb} / \mathrm{Ni}$ core-shells. In particular, at positive values of $H_{\text {ext }}$ and decreasing fields, or vice versa, a pronounced diamagnetic superconducting "dome" is visible, which starts to form at fields far in excess of the critical field of bulk lead. In this regime the side walls of the ferromagnetic shell are almost fully saturated with magnetization $M_{F M}(c . f$, figure $5 \mathrm{~b}$ ) and generate a stray field $H_{F M}$ that opposes the external field, $H_{\text {ext }}$. Hence the local field at the core, which is the vector sum of the stray and applied fields, is lowered to below $H_{c}^{\mathrm{Pb}}$ and superconductivity survives. This compensation effect is clearly visible for all $\mathrm{Pb}-\mathrm{Ni}$ core-shell samples measured (figure 1(a-c)), being strongest in the structure with the longest side length of $5.7 \mu \mathrm{m}$ and weakest in that with the shortest side length of $3.3 \mu \mathrm{m}$. Additional curves are shown for the largest $\mathrm{Pb}-\mathrm{Ni}$ crystal at $T=2 \mathrm{~K} \ll T_{c}^{\mathrm{Pb}}$ (figure 1c, turquoise and blue lines for up and down sweeps respectively). These plots show that at this very low temperature, when the critical field of the core is the highest, the measured magnetization can actually be tuned from para(ferro-)magnetic at high and low fields to absolute diamagnetic near the peak of the superconducting dome.

We also observe superconductivity well above the bulk critical field of the tin core $\left(H_{c}^{\mathrm{Sn}}(2 \mathrm{~K})\right.$ $=214 \mathrm{G}$ ) in the Sn-Ni FSH shown in figure 1d, but the diamagnetic 'dome' is considerably less pronounced than in $\mathrm{Pb}-\mathrm{Ni}$ samples. This is a consequence of the much lower critical field of $\mathrm{Sn}$; the stray fields of the Ni shell only become comparable in magnitude to $H_{c}^{\mathrm{Sn}}$ once it 
has evolved into a significantly demagnetized state. Hence the range of applied fields for which $\left|H_{e x t}+H_{F M}\right|<H_{c}$ is rather narrow and governed more by magnetization reversal of the shell than field compensation effects. Hence, we focus on the properties of $\mathrm{Pb}-\mathrm{Ni}$ core-shell samples in the rest of this paper.

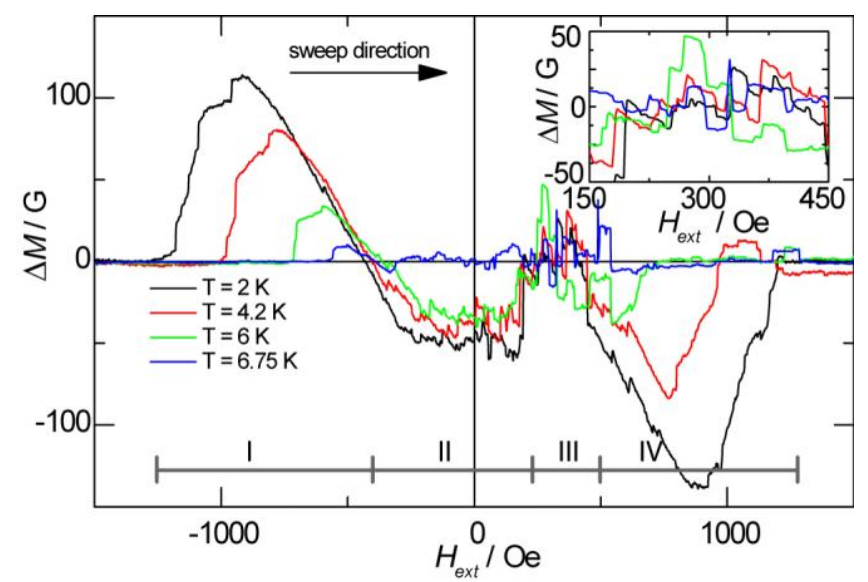

Figure 2.

Plots of the magnetization measured at temperatures $T<T_{c}^{\mathrm{Pb}}$ with the ferromagnetic contribution at $T>T_{c}^{\mathrm{Pb}}$ subtracted. Four different characteristic regions, I - IV, are observed (see text). The inset shows an expanded view of the diamagnetic 'noise' seen in region III.

In order to highlight changes in the magnetization which occur when the core becomes superconducting, figure 2 shows 'difference' traces, $\Delta M$, of the largest $\mathrm{Pb}-\mathrm{Ni}$ triangle sample shown in figure 1 where the measured magnetization data for $T=7.5 \mathrm{~K}>T_{c}^{\mathrm{Pb}}$ have been subtracted from that at various temperatures, $T<T_{c}^{\mathrm{Pb}}$. Four distinct regimes can be identified in these data. For positive applied field sweeps from large negative values $\left(H_{\text {ext }}<-H_{\text {sat }}\right)$ superconductivity first nucleates at the left hand edge of the region labeled I. In this regime the magnetization of the $\mathrm{Ni}$ shell is almost fully saturated with moments pointing downwards (c.f., the corresponding dark grey curve in figure 1 for $T>T_{c}^{\mathrm{Pb}}$ ) and stray fields from the side walls of the Ni shell oppose the direction of the applied field. Superconductivity will first begin to nucleate when $\left|H_{\text {ext }}+H_{F M}\right|<H_{c}(T)$ anywhere in the $\mathrm{Pb}$ core. The nucleated superconducting regions will then continue to grow in size as the applied field is increased and this inequality becomes satisfied in an increasingly larger volume of the core, reaching a 
peak roughly when $\left|H_{e x t}\right| \approx\left|H_{F M}\right|$. For yet more positive applied fields the superconducting volume starts to shrink again because the applied field is too small to fully compensate the $\mathrm{Ni}$ stray fields and collapses completely when $\left|H_{e x t}+H_{F M}\right|>H_{c}(T)$ everywhere in the $\mathrm{Pb}$ core.

At the right hand boundary of region I the magnetization of the Ni shell starts to reverse and the behavior of the core-shell sample becomes much more complex (region II in figure 2). Reversal results in weaker stray fields, $H_{F M}$, in the superconducting core and superconductivity should be restored in some regions of the sample. The surprising zero crossing of $\Delta M$ as a function of $H_{e x t}$ in region II at low temperatures indicates a more paramagnetic net response for $T<T_{c}$. This rather counter-intuitive result must be due to screening of the stray fields of the partially reversed $\mathrm{Ni}$ shell by puddles of superconductivity in the core that actually throws out more flux towards the Hall magnetometer.

The applied field region where the normal state magnetization reversal is steepest is dominated by an irregular series of sharp Barkhausen 'steps' ${ }^{[23]}$ arising from abrupt jumps in the location of domain walls or magnetic vortices between strong pinning sites in the Ni shell. This is labeled region III in figure 2, and the $\Delta M$ data in this regime have been expanded in an inset. We see that each jump in magnetization is accompanied by a correlated jump in the diamagnetic response of the superconducting core. Since local changes in the magnetization structure can either increase or decrease the local stray fields, they can either promote or destroy superconductivity resulting in the non-monotonic diamagnetic "noise" clearly visible in the inset of figure 2.

A further increase in applied field (region IV in figure 2) yields another diamagnetic superconducting 'dome', similar to that seen in region I but of opposite sign. This indicates that the magnetization of the $\mathrm{Ni}$ shell is now almost fully reversed and saturated along the direction of the applied field, and the same compensation mechanisms come in to play as were discussed for region I. As expected, reversing the sweep direction from $H_{\text {ext }}>H_{\max }$ 
results in the same evolution of diamagnetic signal with regions I-IV reflected about the magnetization axis (y-axis).

In order to gain insight into the size and shape of the superconducting regions in the core at various positions on the $M-H$ curve of the $\mathrm{Ni}$ shell, we have performed micromagnetic simulations of our samples using the finite-element solver NMAG. In our micromagnetic model we only consider the magnetic properties of the shell. We set the constant for the exchange coupling equal to $7.2 \cdot 10^{-7} \mathrm{erg} \mathrm{cm}^{-1}$, and the saturation magnetization $M_{\text {sat }}$ equal to 6409 Oe. ${ }^{[24]}$ The inclusion of the magnetocrystalline anisotropy of $\mathrm{Ni}$ is non-trivial due to the polycrystalline character of the Nickel shell and has not been considered here. NMAG discretizes the micromagnetic equations on an unstructured (tetrahedral) mesh. Sufficient accuracy of the method can be ensured by keeping the tetrahedral edge length smaller than the exchange length, $l_{\text {exch }}$. We define a separate, relatively coarse mesh within the core region (with a tetrahedral edge length of about $20 \mathrm{~nm}$ ), since we want to resolve the shell's stray field there. After having relaxed the magnetization of the shell, we can thus probe the stray field at the nodes of the core mesh. NMAG computes the magnetostatic interaction using a hybrid finite element/boundary element method (FEM/BEM), which involves the assembly and storage of a boundary element matrix. We have approximated this by a hierarchical matrix, which, for the presented simulation, reduces its memory footprint from about 94 GB to 1.3 GB. Since state-of-the-art micromagnetic simulations do not yet allow one to model our actual sample sizes, simulation dimensions have been scaled down by a factor of about 10 to make the problem tractable. We have also ignored the screening currents and associated fields due to the superconducting regions of the core since incorporating such effects would require coupled solutions of the micromagnetic and Ginzburg-Landau equations, something which goes well beyond the scope of this work. Despite these approximations we believe our simulations should give reliable qualitative results when the $\mathrm{Ni}$ shell is close to magnetic 
saturation and will predict the onset fields for the nucleation of superconductivity with reasonable accurately.
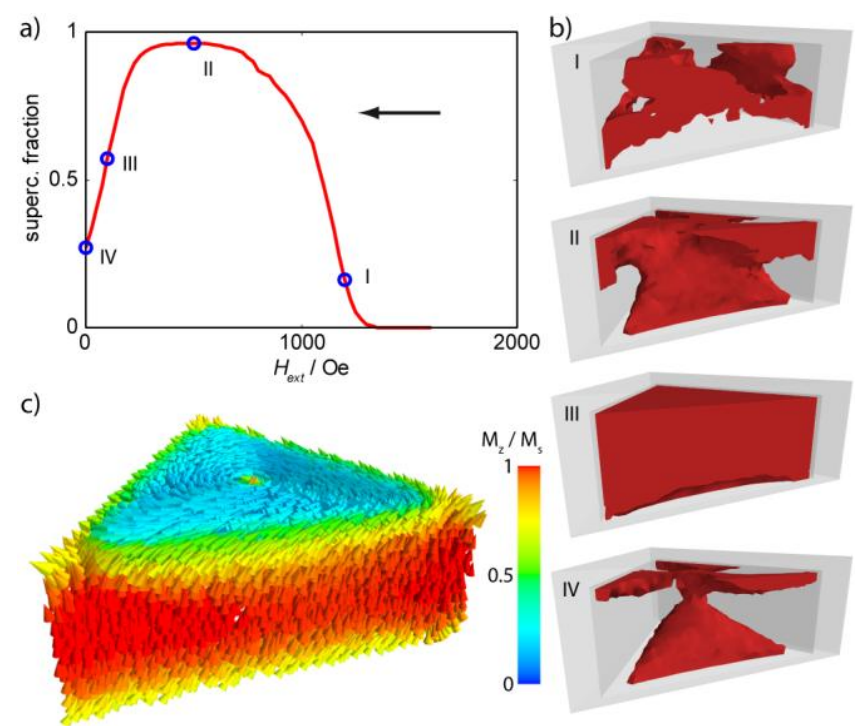

Figure 3.

Micromagnetic simulations of a $\mathrm{Pb}-\mathrm{Ni}$ triangular platelet-shaped core shell sample (see text). (a) The superconducting volume fraction of the core as a function of applied magnetic field. (b) $3 \mathrm{D}$ renderings of the superconducting volumes (dark red) in the core (where $\left|H_{e x t}+H_{F M}\right|<$ $H_{c}{ }^{\mathrm{Pb}}$ ) at the applied fields indicated on (a). (c) 3D vector plot of the magnetization along the zaxis $M_{z} / M_{\text {sat }}$ of the shell at the 'freezing' field of $H_{e x t}=1000$ Oe.

Figure 3 illustrates the results of simulations for a $25 \mathrm{~nm}$ thick Ni shell deposited on the top and sides of a triangular platelet-shaped core of thickness $220 \mathrm{~nm}$ and side-length $570 \mathrm{~nm}$ (c.f., also magnetization data in figure 1c). The plot in figure $3 \mathrm{a}$ shows the calculated superconducting fraction of the core for a range of external magnetic fields (negative sweep direction from $H_{e x t}>H_{m a x}$, where these regions are defined by the inequality $\left|H_{e x t}+H_{F M}\right|<$ $H_{c}$. Bulk $\mathrm{Pb}$ is a type I superconductor with susceptibility $\chi=-1$ in the Meissner state $\left(H<H_{c}\right)$ and we assume that the diamagnetic signal from the core is directly proportional to this superconducting volume fraction. Since domain wall pinning is not present in our micromagnetic model, magnetic reversal starts earlier in simulations than is observed experimentally. To account for this we have 'frozen' the magnetic domain structure at $H_{e x t}=$ 1000 Oe and assumed that it remains unchanged as the field is swept to lower values in figure 3a. We note that our simulations are not at all sensitive to the choice of domain freezing field 
for values larger than 800 Oe. Under these assumptions we see that the estimated superconducting fraction is in good qualitative agreement with the diamagnetic 'dome' seen in this regime in figure 1 . Figure $3 \mathrm{~b}$ shows $3 \mathrm{D}$ plots of the superconducting volume at the various indicated points on figure $3 \mathrm{a}$. We see clearly how superconductivity first nucleates in a ring around the base edges and in three distinct points on the top surface of the core. These regions then expand and join up through the centre until they fill the entire core at the peak of the diamagnetic 'dome'. As the applied field is decreased beyond this point superconductivity starts to be destroyed again from the edges inwards. Figure $3 \mathrm{c}$ shows a 3D vector plot of the magnetization of the shell at $H_{e x t}=1000$ Oe. Note the presence of a magnetic vortex in the centre of the top surface of the Ni shell which is clearly correlated with the nucleation and destruction of superconductivity in the $\mathrm{Pb}$ core in figure $3 \mathrm{~b}$. This observation leads us to speculate that the presence and motion of such vortices is important in determining the detailed evolution of superconductivity in the core.

\section{Discussion}

The onset of superconductivity in a homogeneous bulk sample is uniquely controlled by the external parameters magnetic field, $H_{\text {ext }}$, and temperature, $T$. In contrast, superconductivity in our FSH core-shell structures also has a history-dependence via the magnetization structure of the ferromagnetic shell. This adds a rich new control parameter for the onset of superconductivity and diamagnetism. The criterion for the nucleation of superconductivity in the core is that the ferromagnetic stray fields and applied field must compensate one another so that the vector sum lies below the temperature-dependent critical field, $H_{c}$, locally. Reentrant or field-induced superconductivity have been demonstrated before in essentially planar structures. Our 3D core-shell geometry gives much more control over the local magnetic environment and has allowed us to demonstrate a complete field-driven reversal in the absolute measured magnetic response of the composite structure from para(ferro- 
)magnetic to diamagnetic. In addition, our ability to monitor the total magnetization of the FSH as the $\mathrm{Ni}$ shell is being reversed allows us to directly correlate changes in the magnetization state of the shell with the appearance/disappearance of diamagnetism. A rather compelling example of this is the observation of non-monotonic diamagnetic 'noise' in regime III of figure 2 which is intimately correlated with Barkhausen events in the ferromagnetic shell. Small jumps in the positions of domain walls in the Ni shell lead to local increases/decreases in stray fields and local nucleation/destruction of superconductivity. Hence, this complex diamagnetic signal contains detailed information about the micromagnetic structure of the shell and can be used to inform/test more detailed models of it. Since our structures are grown using electrochemical crystallization it is not possible to include an insulating buffer layer between superconductor and ferromagnet. Hence the superconductor is in direct intimate contact with the ferromagnetic shell and one would expect the suppression of superconductivity near this interface via the proximity effect. However, the proximity effect is only expected to extend about a coherence length $(\xi(0) \approx 80 \mathrm{~nm}$ for $\mathrm{Pb})$ into the surface of the superconducting shell, which is nearly two orders of magnitude smaller than the typical side length of one of our $\mathrm{Pb}$ triangles. Hence the effect would not be expected to have any observable consequences.

The micromagnetic simulations shown in figure 3 reproduce the onset field of the diamagnetic superconducting 'dome' of figure 2 quite well, but its broad inverted U-shaped profile is somewhat different from the more pointed shape observed experimentally. This is a consequence of the simulation of domain wall pinning by freezing the magnetization state of the Ni shell at $H_{e x t}=1000$ Oe while some magnetic relaxation is, in fact, taking place in our measurements in this region. Our simulations also ignore screening currents and fields arising in the diamagnetic core which should be added to the ferromagnetic and external field contributions in a truly self-consistent way in order to calculate the superconducting volumes. 
While this could be achieved in the future by solving coupled micromagnetic and GinzburgLandau equations, it is currently beyond the scope of this work.

Finally we note that several recent theoretical and experimental works ${ }^{[14,25,26]}$ have studied modifications in the magnetization reversal of a ferromagnet in close contact with a superconductor, via changes in the magnetostatic energy arising from screening effects. We have carefully analyzed our data for any sign of this behavior, particularly in regime III of figure 2 where we see the irregular series of abrupt Barkhausen jumps. In practice the field positions of these jumps are not completely reproducible from cycle to cycle, even at $T>T_{c}$. Hence it is not possible to identify any systematic shift in jump positions at $T<T_{c}$ when the core becomes superconducting. However, we cannot rule out that such effects are also at play in our core-shell structures.

\section{Conclusion}

In conclusion, we have demonstrated that it is possible to prepare high quality 3D FSH coreshell structures by electrocrystallization on Boron-doped diamond substrates. We demonstrate that superconductivity survives in these samples up to magnetic fields well in excess of the bulk critical field of the superconducting core due to field compensation effects. For the case of $\mathrm{Pb}-\mathrm{Ni}$ core-shell structures we observe a diamagnetic superconducting 'dome' as a function of applied magnetic field with a maximum at the point where $H_{e x t} \approx-H_{F M}$, where $H_{F M}$ is the stray field of the saturation magnetized $\mathrm{Ni}$ shell at the centre of the $\mathrm{Pb}$ core. For the largest $\mathrm{Pb}-\mathrm{Ni}$ triangles we are actually able to switch the net measured magnetic behavior from absolute para(ferro-)magnetic to diamagnetic by tuning the external magnetic field. Micromagnetic simulations have been used to model the stray field distribution of the ferromagnetic shell and, by mapping the regions of the core where $\left|H_{e x t}+H_{F M}\right|<H_{c}$, predict dome-shaped superconducting volumes as a function of applied magnetic field which are in good qualitative agreement with experiment. Simulations also give valuable insights into the 
size and shape of the superconducting volumes that nucleate in the core under different conditions, and suggest that the motion of magnetic vortices may be important in determining the detailed evolution of superconductivity. We also observe non-monotonic diamagnetic 'noise' in our measurements which is directly correlated with Barkhausen jumps in the shell magnetization and arises from increases or decreases in the local stray fields that either promote or destroy superconductivity.

Hybrid core-shell structures of the type described here could find important applications in advanced magnetic guidance systems or superconducting memory devices as well as for particle detection at very high magnetic fields.

\section{Experimental}

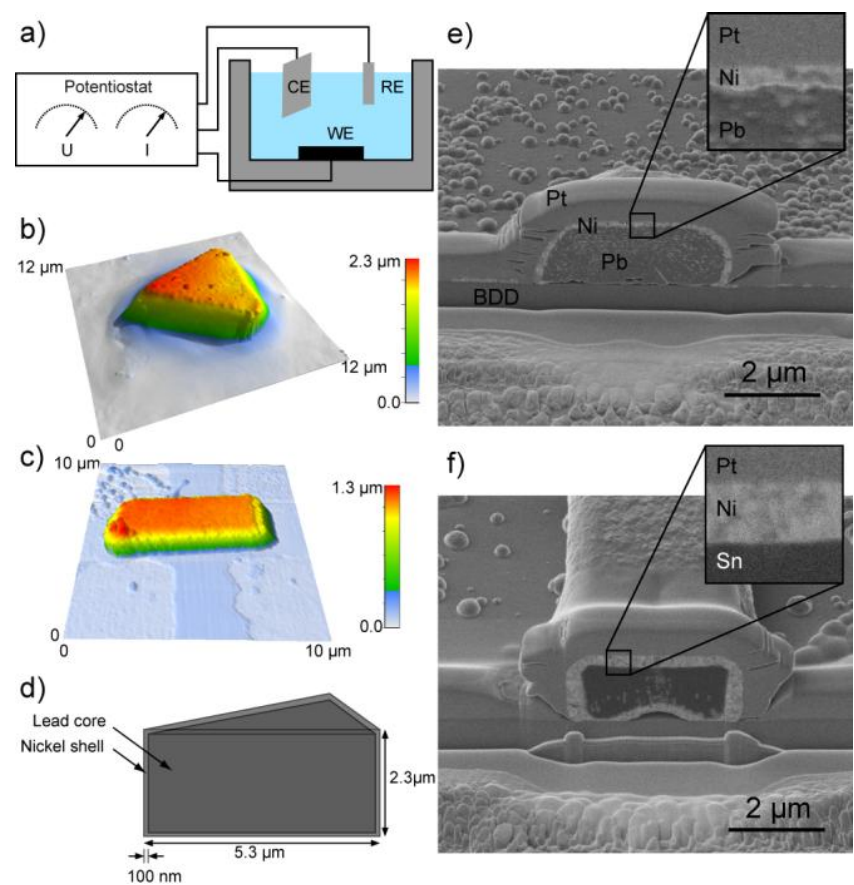

\section{Figure 4.}

a) Schematic diagram of the electrochemical cell used to grow and plate the core-shell samples composed of a counter electrode (CE), a reference electrode (RE) and a working electrode (WE). b) and c) 3D Atomic force microscope images of a triangular $\mathrm{Pb}-\mathrm{Ni}$ platelet and a $\mathrm{Sn}-\mathrm{Ni}$ cuboid respectively. d) Sketch, with dimensions, of the largest $\mathrm{Pb}-\mathrm{Ni}$ triangular prism investigated here. e) and f): FIB milled cross-sections of a $\mathrm{Pb}-\mathrm{Ni}$ and a $\mathrm{Sn}-\mathrm{Ni}$ sample respectively. The side length of the inset expanded images is $500 \mathrm{~nm}$. 
Our experiments have been performed with electrochemically grown tin and lead single crystal cores, which were then electroplated with a nickel shell layer of controllable thickness. This route has many advantages over more conventional lithographic approaches. Not only does it allow us to realize three dimensional core-shell structures, it is also fast and cheap.. Although we are only interested here in individual FSH crystals, each electrodeposition results in a very large number of almost identical structures with very similar properties, ideal for scaling up for future applications.

Superconducting lead microcrystals were prepared from lead(II)nitrate $\left(\mathrm{Pb}\left(\mathrm{NO}_{3}\right)_{2} ; 5-25 \mathrm{mM}\right)$ and boric acid $\left(\mathrm{H}_{3} \mathrm{BO}_{3} ; 100-250 \mathrm{mM}\right)$ solutions made with Milli-Q water $\left(\rho>18.2 \mathrm{M} \Omega \mathrm{cm}^{-1}\right)$. A boron doped diamond (BDD) was used as the working electrode and the crystals were grown for $60 \mathrm{~s}$ at an overpotential of $-800 \mathrm{mV}$ measured versus a $\mathrm{Ag} / \mathrm{AgCl}$ reference electrode. Tin cores were deposited from a tin(II)tetrafluoroborate $(25 \mathrm{mM})$ and fluoroboric acid (100 mM) solution made with Milli-Q water. They were also grown on a BDD electrode at $-780 \mathrm{mV}$ for $30 \mathrm{~s}$. A Watts bath $\left(100 \mathrm{~mm} \mathrm{Ni}\left(\mathrm{SO}_{3} \mathrm{Ni}_{2}\right)_{2}+15 \mathrm{mM} \mathrm{NiCl}_{2}+65 \mathrm{mM} \mathrm{H}_{3} \mathrm{BO}_{3}\right)$ was used to deposit the nickel shell onto all the crystals. Typically the cores were plated for $10 \mathrm{~s}$ at an overpotential of $-1.2 \mathrm{~V}$ versus a $\mathrm{Ag} / \mathrm{AgCl}$ reference electrode. The electrodeposition setup is sketched in figure $4 \mathrm{a}$, while figure $4 \mathrm{~b}$ and $\mathrm{c}$ show two atomic force microscope images of a typical triangular-shaped $\mathrm{Pb} / \mathrm{Ni}$ sample and a square cuboid-shaped $\mathrm{Sn}-\mathrm{Ni}$ sample respectively. Figure $4 \mathrm{~d}$ illustrates the geometry of core and shell in the case of the $\mathrm{Pb}-\mathrm{Ni}$ sample of figure $4 \mathrm{~b}$. The net charge flow during $\mathrm{Ni}$ deposition was used to estimate the thickness of the shell $(\approx 100-300 \mathrm{~nm})$ subject to assumptions about the density and mean surface area of the single crystal cores. In practice very similar conditions were used to grow all 'shells' and the Ni thicknesses are not expected to vary much from structure to structure. Simulations of the saturation magnetization, $M_{\text {sat }}$, measured at the Hall probe under the crystal yield a shell thickness of $\approx 200-300 \mathrm{~nm}$, which is slightly larger but still in good agreement with the estimation from electrochemical parameters and the micrographs in figure $4 \mathrm{e}$ and $\mathrm{f}$. 
Focused ion beam (FIB) sectioning was used to investigate the internal structure of core-shell structures grown in this way. A thin Pt layer was first FIB-deposited on the region of interest in order to protect it while it was being milled. The FIB was then used to slice a cross-section through the core-shell and polish it prior to imaging the exposed surface. The resulting images are shown in figure $4 \mathrm{e}$ and $\mathrm{f}$ for a triangular $\mathrm{Pb}-\mathrm{Ni}$ structure and a cuboid $\mathrm{Sn}-\mathrm{Ni}$ structure respectively. The sharp interface between the tin or lead core and the nickel shell is clearly visible (see insets of figure $4 \mathrm{e}$ and $\mathrm{f}$ ) and there is no evidence at all of alloying or interdiffusion. These images also confirm estimates of the Ni shell thicknesses to be of the order of $\approx 100-300 \mathrm{~nm}$ made on the basis of the electrodeposition parameters.

The low temperature magnetic response of individual FSHs was measured by placing them on one of the $2 \mu \mathrm{m} \times 2 \mu \mathrm{m}$ active elements of a two-dimensional GaAs/AlGaAs (2DEG) heterostructure Hall probe array.[27]

a)

b)

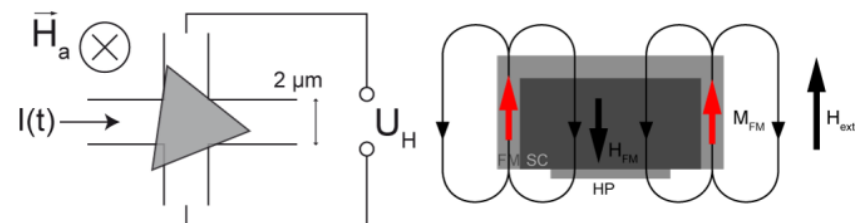

Figure 5.

The measurement geometry of a FSH sample on top of a 2DEG Hall-probe is shown (a) from above and (b) in expanded view from one side. Also indicated on (b) is a simple sketch of the dominant stray magnetic field contribution arising from the vertically magnetized sides of the ferromagnetic shell.

After the successful completion of the core-shell growth steps, individual microcrystals were removed from the BDD electrode under a long focal length optical microscope using a piezoelectric nanomanipulator with a $1 \mu \mathrm{m}$ tungsten tip. They were then individually placed on one of the active elements of a Hall probe array and fixed down with a small amount of low melting temperature paraffin wax. Figure 5a sketches the typical measurement geometry used and shows a triangular core-shell structure on top of one of the active Hall probes at the 
intersection of two $2 \mu \mathrm{m}$ wide wires. Figure $5 \mathrm{~b}$ shows a side view of the same structure where the two-dimensional electron gas forming the Hall effect sensor lies about $80 \mathrm{~nm}$ below the bottom of the mounted core-shell structure. Also shown on this figure is a sketch of the expected stray magnetic field lines from the ferromagnetic shell after saturation magnetization in the upwards direction shown (parallel to $H_{\text {ext }}$ ). Note that the dominant fields in the core arise from moments in the vertical sidewalls of the Ni shell and point in the opposite direction to the externally applied field. The Hall probes were driven with a $20 \mu \mathrm{A} 314 \mathrm{~Hz}$ ac current and the generated Hall voltage was detected using two digital lock-in amplifiers, one of which was used to simultaneously record a reference signal from an 'empty' Hall probe. The two measured Hall voltages were numerically subtracted to obtain a quantity directly proportional to the 'local' magnetization of the sample. The Hall array was mounted on the end of a temperature-controlled sample holder which was inserted into a stainless steel tube containing helium exchange gas, which was in turn immersed in a liquid helium bath. The pressure in the helium bath could be lowered with a rotary pump allowing temperatures down to about $T \approx 2$ $\mathrm{K}$ to be reached. An external magnetic field was applied from a commercial superconducting solenoid attached to the bottom of the sample tube.

Magnetization loops were captured by sweeping the applied field at constant temperature from $H_{\max } \ll<-H_{\text {sat }}$ to $H_{\max } \gg>H_{\text {sat }}$ and vice versa, where $H_{\max }$ is the maximum applied field and $H_{\text {sat }}$ is the saturation field of the ferromagnetic FSH shell. Superconductivity was fully suppressed in all structures at all temperatures at $H_{\max }$. Scans were repeated at various temperatures up to and above the critical temperature $\left(T_{c}\right)$ of the core material. All sweeps at different temperatures were performed using the same values for $H_{\max }$, scan rate and point density to enable an accurate comparison between the scans. This, and the fact that magnetization reversal in the ferromagnetic shell showed no visible temperature dependence for $T<10 \mathrm{~K}$, allows us to subtract the data for $T>T_{c}$ from the data for $T<T_{c}$ in order to 
highlight the contribution of the superconducting core to the magnetization of the entire FSH sample.

\section{Acknowledgements}

We gratefully acknowledge the assistance of Dr P. Heard (IAC, University of Bristol) with FIB sectioning and imaging of core-shell structures.

This work was supported by the Engineering and Physical Sciences Research Council (EPSRC) in the UK under grants EP/E039944/1 and EP/E040063/1, the ESF-NES network and the European Community's Seventh Framework Programme (FP7/2007-2013) under grant agreement no. 233552 (DYNAMAG project).

1. Jaccarino, V. and Peter, M. Phys. Rev. Lett. 9(7), 290-292 Oct (1962).

2. Yang, Z., Lange, M., Volodin, A., Szymczak, R., and Moshchalkov, V. V. Nat Mater 3(11), 793-798 (2004).

3. Rusanov, A. Y., Hesselberth, M., Aarts, J., and Buzdin, A. I. Phys. Rev. Lett. 93(5), 057002 Jul (2004).

4. Lyuksyutov, I. F. and Pokrovsky, V. L. Advances in Physics 54(1), 67-136 (2005).

5. Aladyshkin, A. Y., Silhanek, A. V., Gillijns, W., and Moshchalkov, V.

V.Superconductor Science and Technology 22(5), 053001 (2009).

6. Lange, M., Bael, M. J. V., Bruynseraede, Y., and Moshchalkov, V. V. Phys. Rev. Lett. 90(19), 197006 May (2003).

7. Tagirov, L. R. Phys. Rev. Lett. 83(10), 2058-2061 Sep (1999).

8. Gu, J. Y., You, C.-Y., Jiang, J. S., Pearson, J., Bazaliy, Y. B., and Bader, S. D. Phys. Rev. Lett. 89(26), $267001 \operatorname{Dec}(2002)$. 
9. Nemes, N. M., Visani, C., Leon, C., Garcia-Hernandez, M., Simon, F., Fehér, T., te Velthuis, S. G. E., Hoffmann, A., and Santamaria, J. Applied Physics Letters 97(3), 032501 (2010).

10. Larrea, A., Morales, A., Waysand, G., and Bartolomé, J. Nuclear Instruments and Methods in Physics Research Section A: Accelerators, Spectrometers, Detectors and Associated Equipment 317(3), 541 - 544 (1992).

11. Lin, L. K., Chi, Y. S., Chen, T. M., Shyu, S. G., Huang, J. H., and Lee, S. F. Journal of Applied Physics 105(7), 07D519 (2009).

12. Garif’yanov, N., Goryunov, Y., Mühge, T., Lazar, L., Khaliullin, G., Westerholt, K., Garifullin, I., and Zabel, H. The European Physical Journal B - Condensed Matter and Complex Systems 1, 405-407 (1998). 10.1007/s100510050203.11

13. Bulaevskii, L. N. and Chudnovsky, E. M. Phys. Rev. B 63(1), 012502 Dec (2000).

14. Dubonos, S. V., Geim, A. K., Novoselov, K. S., and Grigorieva, I. V. Phys. Rev. B 65(22), 220513 Jun (2002).

15. Gillijns, W., Aladyshkin, A. Y., Silhanek, A. V., and Moshchalkov, V. V. Phys. Rev. B 76(6), 060503 Aug (2007).

16. Tamegai, T., Nakao, Y., Tsuchiya, Y., and Nakajima, Y. Physica C: Superconductivity 468(15-20), 1308 - 1312 (2008). Proceedings of the 20th International Symposium on Superconductivity (ISS 2007), Proceedings of the 20th International Symposium on Superconductivity (ISS 2007).

17. Miloševic, M. V., Berdiyorov, G. R., and Peeters, F. M. Phys. Rev. Lett. 95(14), 147004 Sep (2005).

18. Golubovic, D. S., Pogosov, W. V., Morelle, M., and Moshchalkov, V. V. EPL (Europhysics Letters) 65(4), 546 (2004).

19. Lange, M., Van Bael, M. J., Silhanek, A. V., and Moshchalkov, V. V. Phys. Rev. B 72(5), 052507 Aug (2005). 
20. Xiao, Z.-L., Han, C. Y., Kwok, W.-K., Wang, H.-H., Welp, U., Wang, J., and Crabtree, G. W. Journal of the American Chemical Society 126(8), 2316-2317 (2004).

21. Müller, A., Dale, S. E. C., Engbarth, M. A., Bending, S. J., and Peter, L. M. CrystEngComm 12(7), 2135-2138 (2010).

22. Dale, S. E. C., Müller, A., Engbarth, M. A., Bending, S. J., and Peter, L. M. in preparation.

23. Barkhausen, H. Z. Phys. 20, 401 (1919).

24. O’Handley, R. C. Modern Magnetic Materials: Principles and Applications. John Wiley \& Sons, (2000).

25. Bergeret, F. S., Volkov, A. F., and Efetov, K. B. Phys. Rev. B 69(17), 174504 May (2004).

26. Fritzsche, J., Kramer, R. B. G., and Moshchalkov, V. V. Phys. Rev. B 79(13), 132501 Apr (2009).

27. Geim, A. K., Dubonos, S. V., Lok, J. G. S., Grigorieva, I. V., Maan, J. C., Hansen, L. T., and Lindelof, P. E. Applied Physics Letters 71(16), 2379- 2381 (1997). 